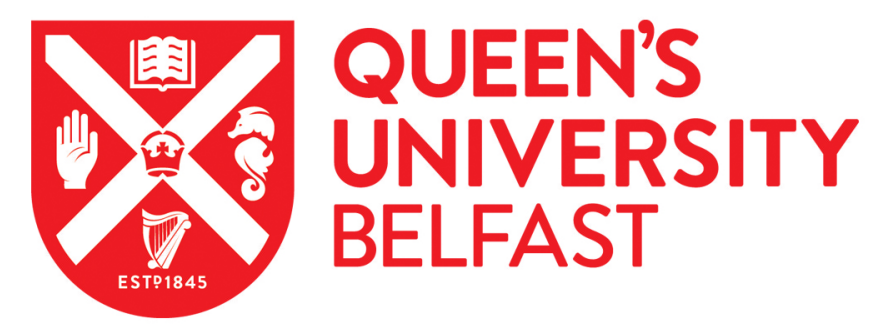

\title{
Prospective Memory Impairment in Chronic Heart Failure: A Replication Study
}

Habota, T., Cameron, J., Thompson, D. R., \& Ski, C. F. (2022). Prospective Memory Impairment in Chronic Heart Failure: A Replication Study. Psychology, Health and Medicine, 27(6), 1277-1285.

https://doi.org/10.1080/13548506.2020.1867317

\section{Published in:}

Psychology, Health and Medicine

\section{Document Version:}

Peer reviewed version

\section{Queen's University Belfast - Research Portal:}

Link to publication record in Queen's University Belfast Research Portal

\section{Publisher rights}

(c) 2020 Informa UK Limited, trading as Taylor \& Francis Group.

This work is made available online in accordance with the publisher's policies. Please refer to any applicable terms of use of the publisher.

\section{General rights}

Copyright for the publications made accessible via the Queen's University Belfast Research Portal is retained by the author(s) and / or other copyright owners and it is a condition of accessing these publications that users recognise and abide by the legal requirements associated with these rights.

Take down policy

The Research Portal is Queen's institutional repository that provides access to Queen's research output. Every effort has been made to ensure that content in the Research Portal does not infringe any person's rights, or applicable UK laws. If you discover content in the Research Portal that you believe breaches copyright or violates any law, please contact openaccess@qub.ac.uk. 
Prospective Memory Impairment in Chronic Heart Failure: A Replication Study

Tina Habota, PhD, ${ }^{1,2}$ Jan Cameron, $\mathrm{PhD},{ }^{3,4}$ David R. Thompson, $\mathrm{PhD},{ }^{4,5}$ Chantal F.

Ski, $\mathrm{PhD}^{4,6}$

${ }^{1}$ Aberdeen Biomedical Imaging Centre, University of Aberdeen, Aberdeen, UK

${ }^{2}$ School of Behavioural and Health Sciences, Australian Catholic University,

Brisbane, Australia

${ }^{3}$ School of Nursing and Midwifery, Monash University, Melbourne Australia

${ }^{4}$ Australian Centre for Heart Health, Melbourne, Australia

${ }^{5}$ School of Nursing and Midwifery, Queen's University Belfast, Belfast, UK

${ }^{6}$ Integrated Care Academy, University of Suffolk, Ipswich, UK

Correspondence and reprint requests to: Tina Habota, School of Behavioural and

Health Sciences, Australian Catholic University, Banyo, QLD 4014, Australia

E-mail: drtinahabota@gmail.com

Word count for abstract -176

Word counts for manuscript -2745 


\begin{abstract}
Cognitive deficits are common in patients with chronic heart failure (HF), but little attention has been given to the investigation of prospective memory (PM) - the ability to execute delayed intentions. Importantly, many aspects of PM are crucial for patient implementation of HF self-care behaviours. Here we provide a replication of our original work involving PM in patients with HF. We compared a group of $51 \mathrm{HF}$ patients to 41 closely matched controls. The primary outcome measure was a laboratory test of PM, Virtual week, which closely simulates PM tasks in daily life. A series of background cognitive tests were also administered. Consistent with our previous work, the HF group had significant PM impairment compared to controls, and these difficulties were generalised across different types of PM tasks. Surprisingly, we did not find any differences on the background cognitive tasks between groups. Compared to controls, the HF group had significant and similar sized deficits on all task parameters assessed (event, time, regular, irregular) of PM function, which likely impacts engagement in HF self-care.
\end{abstract}

Key Words: Chronic heart failure, cognitive function, prospective memory 


\section{Introduction}

Cognitive impairment is common in people with chronic heart failure (HF) and usually results from small vessel brain pathology (Berry et al., 2019). Deficits in retrospective memory, executive function, and psychomotor speed are most common (Cameron, Gallagher, et al., 2016; Cameron, Kure, et al., 2016), and impact engagement in self-care (Cameron, Gallagher, \& Pressler, 2017).

Prospective memory (PM), the ability to remember to perform tasks at some point in the future, is implicated in various important functional behaviours (Hering, Kliegel, Rendell, Craik, \& Rose, 2018). Deficits in PM functioning occur after stroke (Kim, Craik, Luo, \& Ween, 2009) and in HF (Habota et al., 2015), where many selfcare behaviours (e.g., medication adherence, weight monitoring) place a heavy load on intentional cognitive abilities (Habota et al., 2015).

It might be expected that patients with HF will inevitably show deficits in PM functioning given that the most frequent cerebral abnormalities seen in this patient group occur in frontal and temporal neural structures (Alosco \& Hayes, 2015), both of which are strongly implicated in PM performance (Reynolds, West, \& Braver, 2009). Moreover, PM performance requires the application of multiple cognitive elements (McDaniel \& Einstein, 2007; Reynolds et al., 2009), such as retrospective memory and executive control processes (e.g., working memory, attentional control and inhibition, and cognitive initiation). This is an important consideration given that these individual cognitive domains are impaired in HF patients (Alosco et al., 2013; Habota et al., 2015).

Habota et al. (2015) found that compared to a group of demographicallymatched controls, patients with HF had pervasive impairments in PM. To test the reliability and generalisability of these findings, the current study aimed to replicate, 
in a different and larger sample of HF patients, the existence of PM deficits, compared to healthy-matched controls.

\section{Methods}

The study was approved by the Human Research Ethics Committees of St Vincent's Hospital (HREC-A018/14), Alfred Hospital (HREC 531/14) and Australian Catholic University (2014-175V) and completed in accordance with the Helsinki Declaration.

\section{Participants}

Participants included patients with a documented diagnosis of HF with reduced ejection fraction $(<45 \%)$ in the previous two years as confirmed by a cardiologist who were referred via inpatient admission, HF clinic, or cardiac rehabilitation program. Participants were recruited over a 12-month period from July 21, 2015 to July 15, 2016. All patients were actively enrolled in a HF management program in one of three hospitals and able to participate in the study (adequate vision, hearing, and English comprehension). They $(n=51)$ were aged between 20 and 90 years $(M=63.80 ; S D=$ 14.75), predominantly male (69\%) and mostly (78.4\%) with mild HF (New York Heart Association functional classification): left ventricular dysfunction (i.e., 78.4\% systolic) and idiopathic (41.2\%) HF were dominant. Patients had their initial diagnosis for almost three years $(\mathrm{M}=2.92$ years; $\mathrm{SD}=1.94)$ and a moderate level of comorbid disease (Charlson Comorbidity Index mean $=6.21)$.

[Table 1 about here] 
We screened global cognition and excluded two patients who showed signs of potential dementia as operationalized by a score $<82$ on the Addenbrooke's Cognitive Examination - Revised (ACE-R) (Mathuranath, Nestor, Berrios, Rakowicz, \& Hodges, 2000). Patients were also excluded if they resided in a nursing home, experienced a cardiac or cerebrovascular event within the past three months, or had a terminal diagnosis. No patients had a head injury but three had a history of cerebrovascular events.

Healthy controls $(n=41)$ were adults who met the inclusion criteria of: living independently; without a medical diagnosis of HF, or any cardiac event, within the past six months; and adequate cognitive performance on the ACE-R (score $>82$ ). The healthy control group was age and gender matched to those with a diagnosis of HF. Healthy controls were recruited through general community advertising and snowball sampling.

\section{Materials}

Participants completed a battery of cognitive tests that measured crystalised- and fluid-type cognitive task (Cameron et al., 2015). Analyses of groups differences were based on raw scores for all measures, except the National Adult Reading Test (NART), which was converted to a scaled score.

\section{Global cognition}

The $A C E-R$ (Mathuranath et al., 2000), a test of global cognition, was used to identify and exclude participants with possible dementia (scores < 82). Scores range from 0100; higher scores reflect better cognitive function. 


\section{Depression and anxiety}

The Hospital Anxiety and Depression Scale (HADS) (Zigmond \& Snaith, 1983) was used to screen for anxiety and depression, separately ( 7 items each). Higher scores indicate more emotional symptomology.

\section{General intelligence}

The NART (Nelson, 1982) was used as an index of premorbid intelligence.

Standardised IQ scores were calculated using the formula in the administration manual.

\section{Working memory and executive function}

A battery of five tests from the CogState program (Maruff et al., 2009) was used: (i) One Back Task and (ii) Two Back Task to measure working memory: participants were presented with a succession of playing cards on a screen and had to decide if the card displayed was the same as the one presented immediately before (One Back Task) or the same as a card presented two cards previously (Two Back Task): accurate responses represent better working memory on both tasks); (iii) Detection Task to measure psychomotor functioning and speed of processing by responding as quickly as possible to a card flipping over on screen: lower scores indicate better performance; (iv) One Card Learning Task to measure visual learning and memory: participants were presented with a succession of playing cards on screen and asked to indicate if the card currently displayed had been displayed previously; and (v) Identification Task to measure visual attention: participants were asked to decide whether a playing card presented on a screen was red, by pressing the 'yes' or 'no' button: we measured reaction time; lower scores indicate better performance. 


\section{Verbal memory}

The Hopkins Verbal Learning Test - Revised (Brandt, 1991) was used to measure verbal episodic memory. The assessor read aloud a list of 12 words, and participants were required to repeat all the words they could remember, in any order. This procedure was carried out three times. After a 20-min delay filled with other activity, participants were asked to recall as many words as possible from the original list of 12. Higher scores indicate better performance.

\section{Prospective memory}

The brief Virtual Week (Rendell \& Craik, 2000) was used to assess PM. Virtual Week, which has been used extensively with clinical and non-clinical groups and having robust psychometric properties (Rendell \& Henry, 2009) is a computerised board game that simulates a week of daily activities. Participants completed two virtual days, which included eight PM tasks per virtual day. They also completed one practice day to ensure they understood the task. A key distinction of PM task types that was assessed in Virtual Week is between event-based and time-based tasks: the former triggered by event-based cues (e.g., take medication (PM task) at breakfast and dinner (PM cue)), the latter performed at a specific time, or once a specific amount of time has elapsed; for example, taking medication (PM task) at 9AM. Another task distinction assessed was between regular versus irregular PM tasks: the former in Virtual Week frequently repeated and well learned, the latter occurred only once and placed substantially greater demands on retrospective memory.

PM performance was measured as the proportion of missed PM tasks (i.e., tasks that participants had forgotten to perform altogether); and correct PM tasks (i.e., tasks performed within a specified window of time). Responses were coded as a 
proportion ranging from 0-1; higher scores reflected worse PM performance for missed analysis, but the inverse was true for correct analysis(Habota et al., 2015).

\section{Procedure}

At study recruitment, demographic and HF clinical characteristics were collected using patient self-report and medical record review. Neuropsychological testing was conducted during the sub-acute phase, approximately three months from recruitment. The ACE-R was administered, followed by the rest of the test battery in a standardised order. The key test - Virtual Week - was also completed during the same testing session. Participants were tested in a single session (about two hours) in a quiet room at their residence or hospital.

\section{Results}

\section{Participant characteristics}

The two groups did not differ in sex $\left(59 \%\right.$ female vs. $69 \%$ male; $\chi^{2}(1)=.62 ; p=$ .433). The HF group had higher proportions of cardiac risk factors, except for hypercholesterolemia. Groups were matched in age, but the HF group had fewer years of education and lower estimated IQ, and self-reported slightly more symptoms of depression. There were no differences in performance between groups on tests of executive function, working memory, or verbal memory.

\section{[Table 2 about here]}

\section{Analysis of prospective memory accuracy}

The dependent variable, PM performance, was expressed as the proportion of Virtual

Week tasks completed correctly for each of the four categories of tasks: regular event, 
regular time, irregular event, and irregular time. Data were analyzed with a $2 \times 2 \times 2$ mixed analysis of variance (ANOVA) with the between-subjects variable of group (HF, control), and the within-subjects variables of PM task (regular, irregular) and PM cue (event-based, time-based). There was a main effect of group $(F(1,90)=$ $\left.13.45 ; p<.001 ; \eta_{\mathrm{p}}^{2}=.130\right)^{1}$, and no two-way interactions between group and any of the variables (both $F \mathrm{~s} \leq 0.45 ; p \mathrm{~s} \geq .505$ ), but there was a significant three-way interaction $\left(F(1,90)=4.47 ; p=.037 ; \eta_{\mathrm{p}}^{2}=.047\right)$.

The three-way interaction was followed up by two separate $2 \times 2$ (Group $\times$ PM cue) ANOVAs for regular and irregular PM tasks. For regular tasks, PM cue was not a main effect and did not interact with group (both $F \mathrm{~s} \leq 1.02 ; p \mathrm{~s} \geq .314$ ), but group was a main effect, $\left(F(1,90)=8.56 ; p=.004 ; \eta_{\mathrm{p}}^{2}=.087\right)$. The HF group performed worse $(M=.67 ; S D=.34)$ than the control group $(M=.84 ; S D=.27)$ on regular tasks. For irregular tasks, group was a main effect $(F(1,90)=11.01 ; p=.001$; $\left.\eta_{\mathrm{p}}{ }^{2}=.109\right)$ and PM cue was a main effect $\left(F(1,90)=17.50 ; p<.001 ; \eta_{\mathrm{p}}{ }^{2}=.163\right)$, but group and PM cue did not interact $\left(F(1,90)=3.30 ; p=.073 ; \eta_{\mathrm{p}}^{2}=.035\right)$. For irregular tasks, the HF group performed worse $(M=.47 ; S D=.30)$ than the control group $(M=.66 ; S D=.33)$, but all participants performed better on event-based PM tasks $(M=.63 ; S D=.32)$ than time-based PM tasks $(M=.48 ; S D=.33)$.

\section{Analysis of missed prospective memory responses}

The alternative index of PM performance was the proportion of Virtual Week tasks missed for each of the four categories of tasks: regular event, regular time, irregular

\footnotetext{
${ }^{1}$ We also ran sensitivity analyses excluding the three HF patients with a history of a cerebrovascular event and found that the group main effect remained significant $(F=13.33 ; p<.001)$.
} 
event, and irregular time. Data were analyzed with a $2 \times 2 \times 2$ mixed ANOVA with the between-subjects variable of group (HF, control), and the within-subjects variables of PM task (regular, irregular) and PM cue (event-based, time-based). Group did not interact with any of the variables (all $F_{\mathrm{s}} \leq .17 ; p \mathrm{~s} \geq .678$ ) but the main effect of group was significant $\left(F(1,90)=5.67 ; p=.019 ; \eta_{\mathrm{p}}{ }^{2}=.059\right)^{2}$; the HF group missed more responses $(M=.21 ; S D=.28)$ than the control group $(M=.12 ; S D=$ $.22)$.

There was also a main effect of PM task $\left(F(1,90)=15.00 ; p=<.001 ; \eta_{\mathrm{p}}^{2}=\right.$ .14) but not for PM cue, and the two-way interaction between PM task and PM cue was also not significant (all $F \mathrm{~s} \leq .34 ; p \mathrm{~s} \geq .561$ ). The participants overall missed more irregular $(M=.23 ; S D=.26)$ than regular tasks $(M=.11 ; S D=.26)$.

\section{Analysis of shared variance}

Exploratory analyses were conducted to examine the potential role of other variables in the observed group difference on the proportion of missed PM tasks. Four $2 \times 2 \times 2$ mixed analyses of covariance (ANCOVAs) were conducted with the between-subjects variable of group (HF, control), and the within-subjects variable of PM task (regular, irregular) and PM cue (event, time), and one of the following variables entered as a covariate in each ANCOVA: global cognition, premorbid IQ, education, and depression. The dependent variable was the proportion of missed responses. Results showed that the medium effect size $\left(\eta_{\mathrm{p}}^{2}=.06\right)$ dropped to .04 (education), .04 (premorbid IQ), .03 (global cognition), and .05 (depression), but remained the same (.06) for age. Thus, each of these variables individually covaried with the PM deficits

\footnotetext{
${ }^{2}$ We also ran sensitivity analyses excluding the three HF patients with a history of a cerebrovascular event and found that the group main effect remained significant $(F=5.50 ; p=.019)$.
} 
observed in the HF group, but global cognition covaried the strongest. Covarying each of these variables reduced the statistical significance of the group main effect ( $p$ s $>.061)$.

\section{Discussion}

Patients with HF have reduced cognitive function in a number of domains (Cameron et al., 2016; Cameron et al., 2016), but this study is only the second to have investigated PM function in this clinical group, compared to healthy controls. Using a different and larger sample, this replication of Habota et al. (2015) found that patients with HF had significant and similar sized deficits on all task parameters assessed (event, time, regular, irregular), suggesting that the cause of the observed PM impairment involves the prospective component particularly. If HF participants' poor performance was solely the result of retrospective memory failure, PM performance would have been weaker on the irregular tasks compared to the regular tasks. Notably, this sample was younger, had more cumulative years of education, and higher estimated premorbid IQ. Thus, even in this group of HF patients, who were higher functioning, pervasive deficits in PM were evident in the original ANOVA analysis. Our findings are consistent with the PM literature on other clinical groups, such as multiple sclerosis (Rendell, Jensen, \& Henry, 2007) or stroke (Kim et al., 2009), who are affected by localised and diffuse brain pathology. More specifically, despite some variability across different clinical groups, a pattern of consistent deficits in PM, irrespective of PM task demands, is reported.

Surprisingly, we found no differences in performance between HF patients and healthy controls on any of the background cognitive tasks. Our findings suggest that compared to other more general cognitive processes, PM decline might be particularly sensitive to HF-related pathology. We found no differences in performance on 
measures of working memory, executive function, and other aspects of memory, but all aspects of PM function were impaired in this HF group compared to healthy controls, findings consistent with Habota et al. (2015).

Exploratory analyses of the potential influence of key demographic factors showed that estimated premorbid IQ, global cognition, and depression overlapped substantially with the group difference in PM performance, as might be expected. The loss of statistical significance from the original ANOVA analysis may reflect loss of power; thus, we focused on effect sizes instead. The ANCOVA results showed that the effect size reduced by a small amount, but the degree of covariation was not substantial.

A key strength of this study is that participants with HF were thoroughly characterized on demographic, clinical, and cognitive features. Limitations were that whilst both groups were closely matched on age and sex, they were not equally matched, as intended, on other demographic variables known to affect cognitive function. The HF group had fewer years of education, lower estimated IQ, and higher self-reported symptoms of negative affect. Future studies should include a clinical control group matched on aspects of physical and affective functionality. The HF group was relatively high functioning; $79 \%$ had no, or only mild, HF symptoms and we excluded people with indicators of dementia. Further, although symptoms of negative affect were higher in the HF group, the extent of depressive symptoms did not quite meet clinical threshold. These are important considerations because in the wider population of patients with HF, where medical and emotional symptoms are often more severe, and global cognition is often more impacted, PM failures are also likely to be greater. 
The investigation of PM in patients with HF deserves further attention as problems with PM cause more difficulties in daily living than other memory failures (McDaniel \& Einstein, 2007). This is particularly relevant to HF patients due to the numerous and concurrent tasks involved in self-care, such as taking medications, daily weighing, which place a high load on their PM ability.

\section{Acknowledgements}

The authors gratefully acknowledge the contribution of Professor Peter Rendell. The study was funded by a Heart Foundation Vanguard grant (100262) and an Australian Government Collaborative Research Network programme grant (2013000375).

\section{Conflict of interests}

There are no conflicts of interest to declare. 
Table 1. Demographic and clinical characteristics of the HF group

\begin{tabular}{lcc}
\hline & $\%$ & $n$ \\
\hline NYHA classification & 78.4 & 40 \\
Class I and II & 15.7 & 8 \\
Class III and IV & & \\
Heart failure type & 78.4 & 40 \\
Systolic & 21.6 & 11 \\
Diastolic & & \\
Heart failure aetiology & 19.6 & 10 \\
Ischemic & 19.6 & 10 \\
Non-ischemic & 41.2 & 21 \\
Idiopathic & 3.9 & 2 \\
Other & & \\
Prescribed medications & 17.6 & 9 \\
Either on ACEI or ARB & 9.8 & 5 \\
Prescribed diuretic & 7.8 & 4 \\
Prescribed aldosterone antagonist & 17.6 & 9 \\
Prescribed beta blocker & & \\
\hline
\end{tabular}

Notes. ACEI, Angiotensin Converting Enzyme Inhibitor; ARB, Angiotensin II Receptor Antagonists; NYHA, New York Heart Association. 
Table 2. Participant characteristics

\begin{tabular}{|c|c|c|c|c|c|c|c|c|c|}
\hline & \multicolumn{3}{|c|}{ HF group } & \multicolumn{3}{|c|}{ Control group } & \multirow[b]{2}{*}{$\chi^{2}$} & \multirow[b]{2}{*}{$p$} & \\
\hline & $n$ & $\%$ & & $n$ & $\%$ & & & & \\
\hline \multicolumn{10}{|l|}{ Cardiac risk factors (\%) } \\
\hline Hypercholesterolemia & 23 & 45.1 & & 15 & 36.6 & & 0.68 & .410 & \\
\hline Hypertension & 22 & 43.1 & & 3 & 7.3 & & 14.74 & $<.001 * * *$ & \\
\hline Smoking & 31 & 60.8 & & 15 & 36.6 & & 5.32 & $.021 *$ & \\
\hline Diabetes & 24 & 47.1 & & 0 & 0.0 & & 26.10 & $<.001 * * *$ & \\
\hline Obesity & 18 & 35.3 & & 2 & 4.9 & & 12.36 & $<.001 * * *$ & \\
\hline Demographic $(M)$ & & $M$ & $S D$ & $n$ & $M$ & $S D$ & $t$ & $p$ & $d$ \\
\hline Age (years) & 51 & 63.80 & 14.75 & 41 & 64.29 & 9.26 & -0.19 & .847 & 0.04 \\
\hline Education (years) & 51 & 14.04 & 3.17 & 41 & 15.95 & 3.91 & -2.59 & $.011 *$ & 0.54 \\
\hline Estimated IQ (NART) & 50 & 114.42 & 4.99 & 40 & 117.15 & 4.49 & -2.69 & $.009 * *$ & 0.58 \\
\hline \multicolumn{10}{|c|}{ Global cognition and mental health } \\
\hline Global cognition (ACE-R) & 50 & 91.90 & 3.67 & 41 & 94.37 & 3.38 & -3.31 & $<.01 * *$ & 0.70 \\
\hline Anxiety (HADS) & 38 & 5.16 & 3.46 & 41 & 5.39 & 3.26 & -0.31 & .760 & 0.07 \\
\hline Depression (HADS) & 38 & 4.18 & 2.99 & 41 & 2.12 & 1.45 & 3.86 & $<.001 * * *$ & 0.88 \\
\hline \multicolumn{10}{|l|}{ Working memory } \\
\hline One Back Task & 51 & 30.43 & 4.34 & 41 & 30.66 & 1.89 & -0.31 & .758 & 0.07 \\
\hline
\end{tabular}




\begin{tabular}{lccccccccc} 
Two Back Task & 51 & 32.16 & 0.46 & 41 & 32.10 & 0.30 & 0.74 & .461 & 0.15 \\
Executive function & & & & & & & & & \\
$\quad$ Processing speed (Detection Task) & 51 & 35.12 & 0.40 & 41 & 35.22 & 0.57 & -1.02 & .309 & 0.20 \\
$\quad$ Visual learning (One Card Learning Task) & 51 & 61.80 & 7.03 & 41 & 62.78 & 8.69 & -0.60 & .552 & 0.12 \\
$\quad$ Visual attention (Identification Task) & 51 & 30.10 & 0.36 & 41 & 30.12 & 0.51 & -0.26 & .793 & 0.05 \\
$\quad$ Verbal memory (HVLT-R) & & & & & & & & & \\
$\quad$ Immediate recall & 51 & 25.92 & 5.20 & 41 & 27.27 & 6.32 & -1.12 & .265 & 0.23 \\
$\quad$ Delayed recall & 51 & 8.84 & 2.49 & 41 & 9.56 & 2.74 & -1.32 & .192 & 0.28 \\
\hline
\end{tabular}

Notes. Effect sizes: small $=0.2 ;$ medium $=0.5 ;$ large $=0.8$

$* p<.05$

$* * p<.01$

$* * * p<.001$

ACE-R, Addenbrooke's Cognitive Examination - Revised; HADS, Hospital Anxiety Depression Scale; HVLT-R, Hopkins Verbal Learning Test

- Revised, NART, National Adult Reading Test. 


\section{References}

Alosco, M. L., Spitznagel, M., Raz, N., Cohen, R., Sweet, L., Colbert, L., ... Gunstad, J. (2013). Executive dysfunction is independently associated with reduced functional independence in heart failure. Journal of Clinical Nursing, 23, 829836. https://doi.org/10.1111/jocn.12214

Alosco, M. L., \& Hayes, S. M. (2015). Structural brain alterations in heart failure: A review of the literature and implications for risk of Alzheimer's disease. Heart Failure Reviews, 20, 561-571. https://doi.org/10.1007/s10741-015-9488-5

Berry, C., Sidik, N., Pereira, A. C., Ford, T. J., Touyz, R. M., \& Hainsworth, A. H. (2019). Small-vessel disease in the heart and brain: Current knowledge, unmet therapeutic need, and future directions. Journal of the American Heart Association, 8, e011104. doi: 10.1161/JAHA.118.011104.

Brandt, J. (1991). The Hopkins Verbal Learning Test: development of a new memory test with six equivalent forms. Clinical Neuropsychologist, 5, 125-142 https://doi.org/10.1080/13854049108403297

Cameron, J., Gallagher, R., Pressler, S. J., McLennan, S. N., Ski, C. F., Tofler, G., \& Thompson, D. R. (2016). Sensitivity and specificity of a five-minute cognitive screening test in patients with heart failure. Journal of Cardiac Failure, 22, 99-107. https://doi.org/10.1016/j.cardfail.2015.08.343

Cameron, J., Gallagher, R., \& Pressler, S. J. (2017). Detecting and Managing Cognitive Impairment to Improve Engagement in Heart Failure Self-Care. Current Heart Failure Reports, 14, 13-22. https://doi.org/10.1007/s11897017-0317-0

Cameron, J., Kure, C. E., Pressler, S. J., Ski, C. F., Clark, A. M., \& Thompson, D. R. (2016). Diagnostic accuracy of cognitive screening instruments in heart 
failure: A systematic review. Journal of Cardiovascular Nursing, 31, 412-424. doi: 10.1097/JCN.0000000000000285.

Cameron, J., Rendell, P. G., Ski, C. F., Kure, C. E., McLennan, S. N., Rose, N. S., ... Thompson, D. R. (2015). PROspective MEmory Training to improve HEart failUre Self-care (PROMETHEUS): Study protocol for a randomised controlled trial. Trials, 16, 196.

Habota, T., McLennan, S. N., Cameron, J., Henry, J. D., Ski, C. F., Thompson, D. R., \& Rendell, P. G. (2015). Prospective memory impairment in chronic heart failure. Journal of the International Neuropsychological Society, 21, 183-192. https://doi.org/10.1017/S1355617715000119

Hering, A., Kliegel, M., Rendell, P. G., Craik, F. I. M., \& Rose, N. S. (2018). Prospective memory is a key predictor of functional independence in older adults. Journal of the International Neuropsychological Society, 24, 640-645. https://doi.org/10.1017/S1355617718000152

Kim, H. J., Craik, F. I. M., Luo, L., \& Ween, J. E. (2009). Impairments in prospective and retrospective memory following stroke. Neurocase, 15, 145-156. https://doi.org/10.1080/13554790802709039

Maruff, P., Thomas, E., Cysique, L., Brew, B., Collie, A., \& Snyder, P. (2009). Validity of the CogState brief battery: relationship to standardized tests and sensitivity to cognitive impairment in mild traumatic brain injury, schizophrenia, and AIDS dementia complex. Archives of Clinical Neuropsychology, 24, 165-178. https://doi.org/10.1093/arclin/acp010

Mathuranath, P. S., Nestor, P. J., Berrios, G. E., Rakowicz, W., \& Hodges, J. R. (2000). A brief cognitive test battery to differentiate Alzheimer's disease and frontotemporal dementia. Neurology, 55, 1613-1620. 
https://doi.org/10.1212/01.wnl.0000434309.85312.19

McDaniel, M. A., \& Einstein, G. O. (2007). Prospective Memory: An overview and synthesis of an emerging field. Thousand Oaks, CA: Sage Publications.

Nelson, H. E. (1982). National Adult Reading Test (NART): Test Manual. Windsor: NFER.

Rendell, P. G., \& Craik, F. I. M. (2000). Virtual week and actual week: Age-related differences in prospective memory. Applied Cognitive Psychology, 14, S43S62. https://doi.org/10.1002/acp.770

Rendell, P. G., \& Henry, J. D. (2009). A review of Virtual Week for prospective memory assessment: Clinical implications. Brain Impairment, 10, 14-22. https://doi.org/10.1375/brim.10.1.14

Rendell, P.G., Jensen, F., \& Henry, J.D. (2007). Prospective memory in multiple sclerosis. Journal of the International Neuropsychological Society, 13, 410416. doi:10.1017/S1355617707070579

Reynolds, J. R., West, R., \& Braver, T. (2009). Distinct neural circuits support transient and sustained processes in prospective memory and working memory. Cerebral Cortex, 19, 1208-1221. doi:10.1093/cercor/bhn164

Zigmond, A. S., \& Snaith, R. P. (1983). The Hospital Anxiety and Depression Scale. Acta Psychiatrica Scandinavica, 67, 361-370. https://doi.org/10.1111/j.16000447.1983.tb09716.x 